\title{
LA ORGANIZACIÓN DE LOS ACTORES EN EL DESARROLLO TERRITORIAL EL TREN DE ALTA VELOCIDAD EN LA CIUDAD INTERMEDIA
}

\author{
Jaume Feliu Torrent \\ Departament de Geografia, Història i $\mathrm{H}^{\mathrm{a}}$ de l'Art \\ Universitat de Girona
}

\section{RESUMEN}

Si queremos evaluar el grado de desarrollo local que aporta el Tren de Alta Velocidad (TAV) en una ciudad es primordial observar la capacidad de los actores del territorio de organizarse para aprovechar sus recursos endógenos. Mediante la elaboración de una nueva metodología, se propone el estudio del proceso de desarrollo de tres ciudades intermedias con TAV, Lérida (España), Aviñón (Francia) y Novara (Italia). En las conclusiones se ponen de manifiesto ciertos problemas propios de la ciudad intermedia en la ejecución de su proyecto local, básicamente la existencia de una relación multiescalar conflictiva y de una débil red de actores locales. La fragilidad de este contexto urbano es debida a la falta de correctos procesos de gobernanza interna y externa.

Palabras clave: Tren de Alta Velocidad, desarrollo local, actores territoriales, gobernanza.

\section{ABSTRACT}

If we want to evaluate the degree of local development that High-Speed Train (HST) provides in a city it is fundamental to observe the capacity of the actors of territory of organizing themselves to make use of their endogenous resources. Through the elaboration of a new methodology, the study of the process of development of three medium-sized cities with HST, Lérida (Spain), Avignon (France) and Novara (Italy), is suggested. As conclusion certain characteristic problems of intermediate city are shown in the execution of its local project, basically the existence of a troublesome multiscalar relationship and of a weak network of local actors. The fragility of this urban context is owed to the lack of correct processes of internal and external governance.

Key words: High-speed train, local development, territorial actors, governance. 


\section{Introducción}

En un mundo globalizado cada vez más competitivo territorialmente, las ciudades grandes esperan recibir grandes acontecimientos para prosperar, como Juegos Olímpicos, Exposiciones Universales o Regatas Internacionales. Las ciudades intermedias, en cambio, tienen que confiar en otros revulsivos para progresar, como el «Tren de Alta Velocidad» (TAV). Realmente, muchas ciudades medias esperan que esta gran infraestructura de transporte les permita hacer un salto cualitativo considerable en la economía, el urbanismo, la posición territorial y la calidad de vida. El interés por este campo de estudio nos ha llevado a investigar en qué grado el TAV conduce a procesos de desarrollo local.

Entendemos desarrollo local como el proceso de aumento y reproducción a largo plazo de los recursos locales, tanto materiales como inmateriales, a partir de la implicación de la colectividad (Feliu, 2005). Asimismo, el desarrollo local presupone la activación de factores explícitos e implícitos. Los elementos explícitos serían aquellas características de la ciudad y de su territorio imprescindibles para el desarrollo, que se pueden describir, enumerar y detallar objetivamente «desde fuera», desde un punto de vista exterior a la ciudad. En el caso del TAV, por ejemplo, podemos determinar que la existencia de un importante contingente de población cualificada, de un sector económico potente o de un patrimonio cultural significativo son factores de éxito para la nueva infraestructura. Pero estos factores no son los únicos a tener en cuenta. Son primordiales también los factores implícitos, aquéllos que se gestan «desde dentro», por parte de los agentes del territorio y su relación con los recursos. Son factores difíciles de sistematizar y que acaban de configurar la particularidad de cada contexto urbano, aquello que algunos llaman «código genético» de cada ciudad (Rabin, 2002). Estos factores implícitos se establecen en la relación entre los actores locales y en el pacto que supone el proyecto local de desarrollo.

Es precisamente el estudio de estos últimos factores lo que se quiere mostrar en el texto que sigue. Si en la investigación desarrollada en la tesis doctoral (Feliu, 2005, 2006b) se analizaban tanto los factores explícitos como los implícitos, en este texto se profundizará especialmente en los segundos, los que hacen una referencia más directa a los procesos de organización de los actores territoriales en relación con el TAV.

El TAV es un invento francés desarrollado entre los años sesenta y setenta del siglo XX. El gobierno francés defendía la creación de un nuevo sistema de transporte rápido y eficiente para unir las principales ciudades del país. Por otro lado, quería aprovechar también el sistema ferroviario existente para evitar los importantes gastos que se podían ocasionar. De esta manera nació el Train à Grande Vitesse (TGV), un tren que circularía a más de $250 \mathrm{~km} / \mathrm{h}$ por las vías ferroviarias nuevas y a menos velocidad por las convencionales: sería como «Clark Kent en la vía convencional, y Superman en las vías especiales» (Lolive, 1999). La primera línea TGV se inauguró en 1981 entre París y Lyón. En poco tiempo se demostró que era muy rentable y que se podía extender hacia otras direcciones, siempre partiendo de París. Viendo los éxitos franceses, otros países europeos se añadieron a la aventura de la Alta Velocidad: Italia, Alemania, Holanda, Reino Unido y, en 1992, España. La red de TAV se consideró desde la Comisión Europea como una Red Trans-Europea de Transporte.

A medida que se iba extendiendo la red TAV por Europa se hacía evidente la necesidad de conectar ciudades cada vez más pequeñas, aquéllas que se situaban en el recorrido entre las ciudades grandes. Al principio, estas estaciones se emplazaban en la periferia, pero las ciudades medias pedían estaciones más céntricas, urbanas. Los gobernantes de estas ciudades creían que el TAV podría provocar procesos de desarrollo económico y social. Es decir, que podía comportar «efectos positivos» en el territorio. 
En la actualidad, transcurridos unos años, podemos hacer balance de los efectos socioeconómicos de esta infraestructura de transporte en las ciudades intermedias. Los estudiosos que han trabajado en el tema no son demasiado optimistas al respecto (Martí-Henneberg, 2000; Bellet, 2000; Rabin, 2003; Plassard, 2003; Feliu, 2006a). A partir del estudio de diversas ciudades medias como Mâcon, Montchanin, Valence, Aviñón, Le Mans, Mannheim, Utrecht, Ciudad Real o Puertollano, los autores determinan que el TAV no aporta, por sí solo, un crecimiento económico de la ciudad. En todo caso, el TAV acelera aquellos procesos socioeconómicos y territoriales que ya se estaban produciendo antes de su construcción. El TAV, por lo tanto, «mueve aquello que ya se movía», y difícilmente provoca automáticamente unos nuevos efectos en la estructura económica y social de las ciudades.

En relación a esto, muchos autores ya habían criticado el concepto de «efecto» socioeconómico del Tren de Alta Velocidad (Offner, 1993; Plassard, 1997, 2003; Joignaux, 1997; Burmeister y Colletis-Wahl, 1997; Governa, 2001; Miralles 2002...). Los efectos de una infraestructura suelen ser descritos como efectos directos y efectos indirectos. Los primeros suceden a corto plazo, y comportan una mayor movilidad de la población, una mayor actividad de la estación o un aumento en las expectativas de la ciudad. Suelen producirse de manera parecida en todas las ciudades estudiadas. En cambio, los efectos indirectos, los que se son producidos a medio y largo plazo, suelen ser diferentes según cada ciudad. En unos casos, la estación ha comportado un aumento del precio del suelo en los espacios limítrofes, en otros no. En algunos lugares, la nueva infraestructura ha comportado el cambio de uso de residencia a terciario (oficinas), y en otros no. La conclusión acaba siendo que las grandes infraestructuras en general, y el TAV en particular, no generan los mismos efectos socioeconómicos y territoriales en las diversas ciudades. Es decir, que los «efectos» del TAV no son previsibles en términos de relación causa-efecto.

Este hecho pone de manifiesto que el territorio es un fenómeno complejo. Está formado por lugares, ciudades, regiones, que se han ido configurando históricamente a través de las relaciones sociales y de sus recursos materiales e inmateriales, y responden de manera diferente a los estímulos externos. Como explica el reconocido geógrafo italiano Giuseppe Dematteis (1985), «la tierra se convierte en territorio cuando es medio de comunicaciones, objeto de trabajo, de producciones, de intercambios, de cooperaciones». Pensado de esta manera, el territorio no se puede observar como un espacio abstracto, simple y previsible donde se producen unas relaciones deterministas de causa-efecto, como las que se describen muchas veces con el Tren de Alta Velocidad. Hay que pensarlo de otra manera, por ejemplo, como un sistema complejo.

Efectivamente, una forma de abordar el estudio de la ciudad como fenómeno complejo, y su relación con el TAV, es la teoría sistémica (Maturana y Varela, 1984; Conti, 1996). Podemos ver la ciudad como un sistema complejo, como un conjunto de sujetos y elementos que interaccionan entre sí. Y podemos ver el TAV también como un sistema de transporte. La relación entre los dos sistemas no será, por lo tanto, de causa-efecto, sino de adaptación mutua («acoplamiento estructural»). El sistema de transporte incidirá en la ciudad, pero no determinará su comportamiento.

Así pues, ¿cuáles son los elementos que condicionan que en una ciudad pueda producirse un proceso de desarrollo local? Muchos autores creen que la respuesta está en los sujetos, en los agentes económicos y sociales de la ciudad (Berg y Pol, 1998, Pol, 2002; Plassard, 2003). Si los agentes locales —el gobierno de la ciudad, las empresas, la Universidad, la Cámara de Comercio, las asociaciones de empresarios, culturales, ambientales, etc.- se organizan adecuadamente y crean estrategias conjuntas para aprovechar las ventajas que proporciona el TAV, se producirán mejoras económicas. Si no se crean estrategias, no se producirán los efectos esperados. Es más, para que se produzca un verdadero desarrollo 
local, es decir, el aprovechamiento de los recursos materiales e inmateriales propios de la ciudad, es necesario que el TAV sea percibido como un recurso endógeno para la ciudad. Es necesario que los agentes locales lo entiendan como una forma de potenciar sus propios recursos (Pucci, 1996; Governa, 2001). Si no es así, los únicos que aprovecharán la nueva infraestructura serán unos pocos agentes, muchas veces externos.

Todas estas reflexiones desplazan el debate del desarrollo del TAV hacia la arena política. Es decir, que uno de los elementos más importantes para el desarrollo local será la planificación. Más concretamente, cómo se organizan los agentes locales para planificar estratégicamente el aprovechamiento del TAV, y cómo participan los agentes locales en la planificación a nivel estatal del trazado y la localización de la estación en su ciudad. Por lo tanto, tendremos que determinar cuál es la gestión de la red interna de actores locales y cuál es la dinámica de gobierno multinivel en la planificación estatal y regional.

Todo aconseja que para estudiar el grado de desarrollo que aporta el TAV en una ciudad media se debe construir una nueva metodología que tenga en cuenta varios elementos. En primer lugar, se tendrán que analizar los condicionantes de tipo técnico, como cuál es la localización de la estación —céntrica, periférica, semiperiférica-, cuáles son las características urbanas de la ciudad y del barrio de la estación, cuáles son las potencialidades económicas de la ciudad. En segundo lugar, habrá que estudiar cómo se han organizado los actores locales y qué dinámica de planificación se ha dado entre los diferentes niveles de gobierno.

Este último ejercicio de análisis y diagnosis, el de la organización de los actores territoriales, es el que se ha experimentado en esta investigación; y se ha querido realizar para tres ciudades medias de países diferentes. Las ciudades escogidas han sido Lérida, Avignon y Novara, situadas respectivamente en España, Francia e Italia. Las tres ciudades son consideradas intermedias por varias razones. En primer lugar, por la dimensión: son ciudades de talla media, alrededor de cien mil habitantes. En segundo lugar, por las funciones: son intermediarias entre la gran ciudad y el territorio. Finalmente, porque tienen una identidad de capitalidad, solidez institucional y pasado histórico, que las ha configurado como centro de servicios y equipamientos de un territorio. Otro elemento común de las tres ciudades escogidas es que se localizan en el Arco Mediterráneo europeo, un espacio dinámico y en vías de crecimiento.

En definitiva, se ha querido analizar cuáles son las problemáticas que afectan específicamente a las ciudades intermedias en relación a la organización de los actores implicados en la planificación del TAV. Por este motivo se ha elaborado una lectura transversal de los tres casos estudiados y se han señalado los campos donde aparecen los problemas de organización de las ciudades intermedias.

\section{Metodología}

\subsection{Metodología de Berg y Pol}

Para elaborar la propuesta metodológica es imprescindible remitirnos a algunos trabajos de base. Nos podemos fijar, en primer lugar, en el interesante trabajo de Berg y Pol (1998) donde se analizan 14 áreas urbanas de ciudades medias europeas y que nos muestra aquellos elementos determinantes para el desarrollo de la ciudad a partir del TAV. La metodología que utilizan los autores es doble. Primeramente obtienen información de las entrevistas realizadas a personalidades clave de cada ciudad, como autoridades locales y regionales, cámaras de comercio, etc., para adquirir información de valor cualitativo. Después, realizan un análisis de cada ciudad siguiendo un esquema que intenta abarcar la complejidad de la ciudad, un 
esquema que sirve para hacer un «análisis integral». Los criterios que se consideran para el análisis (tal como se ve en la figura 1) son seis: Accesibilidad, Potencial económico, Calidad del medio ambiente, Equilibrio de la distribución espacial de las actividades, Equilibrio de la distribución social de los efectos y Capacidad de organización.

FIGURA 1. Análisis integral del TAV

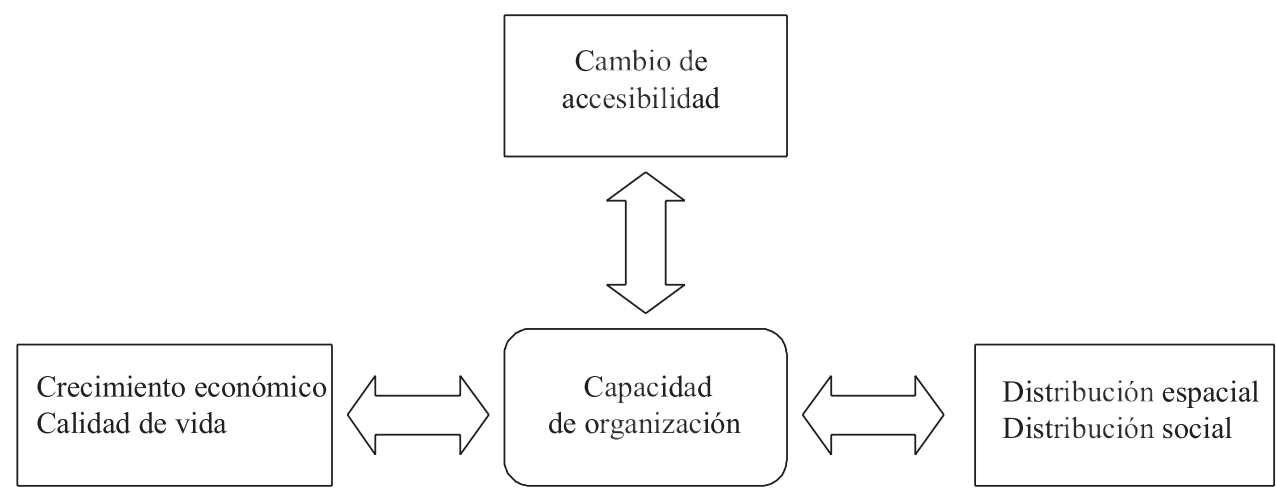

Fuente: Berg y Pol, 1998, p. 9

De los aspectos estudiados por Berg y Pol (1998), es de especial relevancia el estudio de la «capacidad de organización», un condicionante substancial para la buena implementación del TAV en un sistema urbano. Se la considera primordial porque recoge los intereses de los actores implicados y permite la utilización óptima del conocimiento, trabajo y capital disponibles. La capacidad de organización se define como:

«... la habilidad de aquellos responsables para solucionar un problema convocando a los partners implicados (públicos y privados, internos y externos), para generar conjuntamente nuevas ideas y formular e implementar una política que responda a los desarrollos fundamentales y crear las condiciones para el crecimiento económico sostenible.» (Berg, Der Meer y Pol, 2003, p. 1.961)

Esta definición tiene muchos aspectos coincidentes con el concepto de «gobernanza» urbana y territorial definido por dos autores de la siguiente forma:

«un proceso de coordinación de actores, de grupos sociales, de instituciones para atender los propios hitos discutidos y definidos colectivamente dentro de ambientes fragmentados, inciertos.» (Bagnasco y Le Galès, 1997, p. 38); o «una modalidad de coordinación de las dinámicas económicas y sociales que se basa en la implicación y la participación de una multiplicidad de actores» (Governa, 2002).

El análisis de la capacidad de organización se basa en la diferenciación de los tres componentes que conforman las condiciones para el desarrollo económico: Hardware, software y «orgware» (Porter, 1990, citado en Berg y Pol, 1997). Hardware se refiere a los factores tangibles de localización, incluyendo trabajo, tierra, infraestructuras y capital. Software se 
refiere a los factores de calidad de vida intangibles, cualitativos. Y orgware se refiere a la gestión del hardware y el software.

Además, Berg y Pol (1997) — esta vez a partir de un estudio de 8 ciudades europeas-, elaboran una metodología para analizar la capacidad de organización de las ciudades a partir de varios elementos, tal como se puede observar en la siguiente figura:

Figura 2. Marco teórico de la capacidad de organización

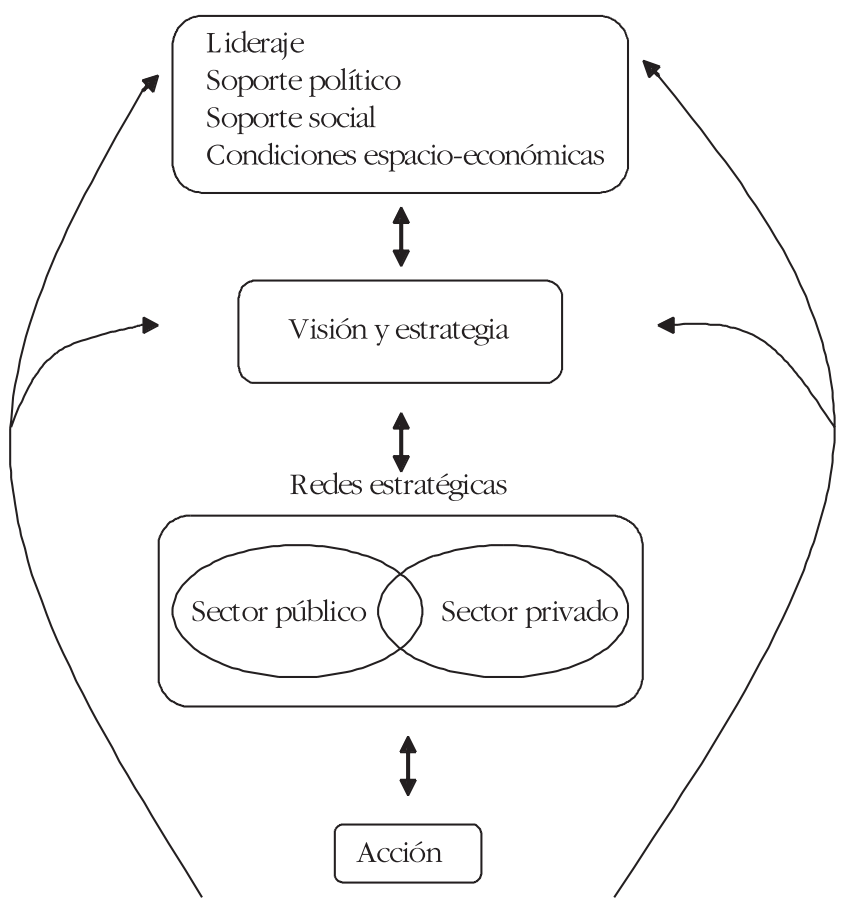

Fuente: Berg y Pol, 1997, p. 260

En la organización de la administración se evalúan varios criterios como la adecuación de la escala espacial de gobierno o las diferentes competencias de los niveles administrativos. Una de las conclusiones del trabajo es que la cooperación entre los municipios implicados es una condición necesaria para el éxito del modelo urbano.

En las redes estratégicas se analizan las alianzas, los partenariados, entre los actores públicos y privados, ya que la gestión urbana y las acciones de gobernanza no son sólo una cuestión de la administración pública formal. Sin embargo, se detecta que la gestión de la red tiene ciertas limitaciones respecto de su continuidad temporal, y por este motivo se considera importante que las instituciones formales tengan un papel destacado.

También se analiza si hay algún actor que ejerce un liderazgo claro dentro de la red de actores, normalmente dentro de las organizaciones administrativas, ya que una falta de liderazgo comporta dificultades en la consecución de los resultados esperados. En algunos casos se ha producido una sucesión de líderes involucrados dentro de un mismo proyecto. 
El análisis de las condiciones espacio-económicas es revelador de la capacidad de la ciudad para crear un nuevo proyecto económico a partir de un acontecimiento como la llegada del TAV. Los autores parten de la hipótesis que las debilidades espacio-económicas pueden inducir a los actores a colaborar. Por lo tanto, sin el reconocimiento de los problemas de la ciudad o la región la creación de capacidad de organización es más problemática.

Otro de los aspectos estudiados es la visión, aquella capacidad de dar un sentido de conjunto, integrado y estratégico a todos los aspectos de las políticas urbanas. Sin visión la capacidad de organización es una utopía, un «misil sin guía».

El soporte político, especialmente financiero, de las administraciones superiores a los gobiernos locales es una condición necesaria para la capacidad de organización. Asimismo, el soporte social, a veces subestimado, es también importante. En este caso es interesante ver si se ha realizado alguna estrategia de comunicación desde el comienzo del proyecto para atraer el apoyo y la implicación de la población.

Finalmente, el último aspecto estudiado es la acción («performance») y coherencia del conjunto del proyecto, para ver hasta qué punto se ha construido un sistema dinámico de relaciones entre los diferentes agentes, a lo largo de las diferentes etapas de gestación del proyecto.

\subsection{Metodología de Dematteis}

Un segundo trabajo de referencia para elaborar la metodología de estudio es el realizado por Dematteis y el grupo de investigación de Eupolis (Dematteis, 2003). La metodología se basa en todo un cuerpo teórico que se dirige al análisis de los procesos de desarrollo local. Dos conceptos clave en la teoría son milieu y Sistema Local Territorial (SLoT). Desde un paradigma «territorialista» (Magnaghi, 1990, 2000), milieu es definido como el:

«conjunto permanente de caracteres socioculturales sedimentados en una cierta área geográfica a través de la evolución histórica de relaciones entre los sujetos, al mismo tiempo en relación con las modalidades de utilización de los ecosistemas naturales locales» (Dematteis, 1994, p. 15)

El milieu estaría caracterizado tanto por condiciones naturales como culturales (tradición, atmósfera industrial, conocimiento, etc.) depositadas a lo largo de la historia. El milieu es difícilmente objectivable y no es definible a priori, en tanto que es un conjunto de condiciones que a menudo no nos son visibles, no nos aparecen, hasta que se convierten en un recurso y son utilizadas por una sociedad local. Los sujetos sociales de un mismo territorio se vinculan entre ellos para sacar partido, para poner en valor algunos aspectos de su milieu. Se establecen, por lo tanto, dos tipos de relaciones: unas horizontales, entre miembros de la sociedad local, y otras verticales, entre los actores locales y los componentes «territoriales» del milieu. Así pues, la capacidad de una colectividad territorial de generar procesos de desarrollo dependería, en primer lugar, del grueso y características de su milieu, pero sobre todo de la capacidad para detectar qué elementos del milieu pueden convertirse en recurso.

El segundo concepto proviene, entre otros, de las teorías de los sistemas complejos (Maturana y Varela, 1989; Foerster, 1985). La característica principal del sistema autopoiético de Maturana i Varela es la capacidad de reproducir sus especificidades con sus propias reglas, o sea, la autopoiesis. Pero lo más interesante de la teoría es la descripción de cómo esto se produce. En cualquier sistema autopoiético se realizan dos estrategias paralelas: una de mantenimiento y otra de cambio. Por un lado, el sistema mantiene su «organización»a 
través de lo que llaman «cierre operativo». De esta manera las características del sistema se mantienen invariadas. Por el otro, el sistema se adapta a los cambios exteriores cambiando su «estructura», es decir, a través de los procesos de «apertura» a las características de su medio y de los otros sistemas. Y esta adaptación a los cambios externos se produce a través de la adaptación de las estructuras, el «acoplamiento estructural». Este proceso se considera básico para el funcionamiento del SLoT, definido como:

«una agregación de sujetos en interacción recíproca los cuales, en función de las relaciones específicas que tienen en un cierto ambiente o milieu local, se comportan, en ciertas circunstancias, como un sujeto colectivo.» (Dematteis, 1993, citado por Governa, 1997, p. 40).

Para la creación de un SLoT se cumplen dos procesos. Primeramente, en un territorio aparece un «sistema local», un agregado de sujetos que se comportan como un sujeto colectivo. Los sujetos de este sistema, a lo largo del tiempo, configuran un «milieu social», fruto de una organización y de un aprendizaje colectivo, a través de sus interacciones estables. Es una entidad autopoiética, cuya finalidad es auto-reproducirse. En segundo lugar, el sistema puede producir una serie de relaciones estables con su territorio, con su ambiente local, formando así un «milieu territorial». La relación del sistema con el ambiente local y el milieu territorial se lleva a cabo a través de «relaciones verticales», que se combinan con las «relaciones horizontales» entre los actores locales y supralocales. La doble lógica permite evitar el determinismo ambiental (sólo de vínculos verticales) y el determinismo espacial (sólo de vínculos horizontales).

A partir de estos conceptos se ha configurado una metodología llamada «tabla de análisis de un SLoT» (Dematteis, 2003), que se organiza en tres bloques principales, con los consiguientes subapartados:

1. Análisis de los proyectos y de las acciones

a. Evolución de la proyectualidad

b. Conjunto de proyectos

c. Objetivos del proyecto

d. Sujetos activos, su rol y acción

e. Capacidad innovadora

f. Relación con el nivel supralocal

g. Relación con el territorio

2. Características del SLoT

a. Las redes locales de los sujetos

b. Las conexiones con el exterior

c. Las relaciones con el milieu

d. Las potencialidades territoriales específicas

e. La cohesión funcional y la estabilidad del SLoT

3. Evaluación

a. Coherencia entre proyectos y recursos potenciales disponibles

b. Valor Añadido Territorial de las acciones y de los proyectos

c. Sostenibilidad territorial de las relaciones con el milieu 


\subsection{Metodología propuesta}

Así pues, la metodología propuesta finalmente se sintetiza en el esquema que se describe a continuación. Para su elaboración, en primer lugar, se recoge información de tipo cuantitativo y cualitativo de la ciudad. Respecto a la información cualitativa se considera importante la realización de entrevistas en profundidad a los expertos locales en este campo de estudio. En segundo lugar, se ordena la información siguiendo la primera parte del esquema, que recoge las dos formas de observar las características de la ciudad: las características técnicas de la intervención y las características organizativas de los agentes. Los apartados de este análisis se subdividen tal como se observa a continuación:

\section{Características técnicas de la intervención}

Aspectos del sistema de transporte

Posición territorial de la ciudad a partir del TAV

Características de la estación TAV

Aspectos de la intervención urbanística

Modelo urbanístico a escala municipal/urbana

Modelo urbanístico a escala de la estación

Aspectos de la planificación económica

Diferentes proyectos económicos de la ciudad relacionados con el TAV

\section{Características organizativas de los agentes}

Capacidad y tipología de estructuración externa

Los agentes supralocales y sus proyectos

Grado de conflictividad y cooperación en las relaciones de los agentes locales y

Supralocales

Capacidad y tipología de organización interna

Capacidad de creación de un proyecto de ciudad a partir del TAV

Agentes que participan en el proyecto local y su dinámica

Ámbito territorial de los proyectos

En tercer y último lugar, se realiza una lectura conclusiva que evalúa el proceso y el producto de la implantación del TAV en la ciudad a partir del Sistema Local Territorial (SLoT) como instrumento de análisis. Se sigue el siguiente esquema:

\section{Evaluación del proceso}

Red de actores locales

Ámbito territorial de la red de actores

Cohesión funcional

A continuación, se describen los apartados correspondientes exclusivamente a las características organizativas de los agentes, los que afectan en concreto al tema de este texto.

Los agentes supralocales y sus proyectos: se observan las funciones dadas al TAV a diversas escalas, partiendo de la hipótesis que la gradación de los proyectos Estatal-Regional-Local es también una gradación de proyectos donde predomina la funcionalidad de la infraestructura («interés general»), en un extremo, o la capacidad de transformación local («interés particular»), en el otro. Se describirán los proyectos del Estado, el de la Región y los intereses de las ciudades mayores, y el de las empresas gestoras de la nueva infraestructura. 
Grado de conflictividad y cooperación en las relaciones de los agentes locales y supralocales: se realiza un repaso de las dinámicas decisiones de la implantación del TAV en la ciudad, y hasta qué punto han comportado grados de conflicto o cooperación entre los diversos agentes implicados. Se parte de la hipótesis que los procesos de gobernanza pueden ser más beneficiosos para la ciudad que los de gobierno (government). Habrá que ver cuál es la respuesta (reactiva, positiva) desde el primer momento que se plantea construir la estación TAV, y como va evolucionando a medida que avanza el proceso de planificación.

Capacidad de creación de un proyecto de ciudad a partir del TAV: interesa ver los cambios entre el proyecto anterior y el nuevo para resaltar las nuevas aportaciones que han aparecido con el TAV. Hay que conocer si la llegada del TAV ha provocado nuevas expectativas en la ciudad para aprovechar recursos que hasta aquel momento no se habían explotado, o nuevos espacios que no tenían valor hasta este momento. Hay que ver si el TAV ha dado una nueva dirección e impulso al proyecto local y como éste se adapta al nuevo contexto territorial de la ciudad. Será importante determinar también qué instrumentos de planificación urbanística, estratégica, territorial se han utilizado.

Agentes que participan en el proyecto local y su dinámica: se describirán qué actores han construido el proyecto de ciudad y cómo lo han hecho. Hay que observar, en primer lugar, las características de los agentes locales, como cuál es su ámbito y capacidad de acción temática y territorial. En segundo lugar, hay que ver las dinámicas que se han producido: qué conflictos y cooperaciones internas, cuál es la estructura de organización interna, cuáles son los principales agentes de la red de actores, qué diversidad de actores ha activado, quién ostenta el liderazgo, las visiones de futuro, el apoyo social, participación, apoyo político, etc.

Ámbito territorial de los proyectos: en el último apartado se observará en qué escala territorial se están produciendo los proyectos de transformación relacionados con el TAV, en qué escala se está produciendo el acuerdo entre los diversos agentes locales, que puede ser a escala municipal, de área urbana, territorial, etc. Este hecho nos ayudará a determinar el alcance del SLoT.

\section{Características del proceso}

La evaluación del proceso de implantación del TAV se efectuará a partir de la descripción de la dinámica del SLoT, con la subdivisión en las siguientes partes: red de actores locales, ámbito territorial de la red de actores y cohesión del sistema.

Red de actores locales: se describen cuáles son las características de los sujetos del SLoT, qué particularidades tiene la red de actores que se ha formado a partir del proyecto o proyectos alrededor del TAV. Se deberá estudiar qué actores forman la red, quién ha ejercido el rol de visión, lideraje, qué relación existe entre actores, qué instrumentos han permitido y favorecido la creación de la red, si se da cooperación o conflictividad, si hay participación de agentes externos.

Ámbito territorial de la red de actores: se quiere determinar cuál es el ámbito territorial del SLoT que se está describiendo. Con la información anterior sobre los ámbitos territoriales de los proyectos se determinará qué ámbito tiene el SLoT, municipal, área urbana, otra escala, cuestión útil para saber dónde se pueden producir procesos de desarrollo local.

Cohesión funcional del sistema: se interpretan cuáles son las características de funcionamiento del sistema, tanto desde el punto de vista interno — su organización-, como desde el punto de vista externo - la adaptación de su estructura a las influencias externas. Analizaremos, por lo tanto, qué grado de cierre y de obertura tiene el SLoT estudiado. Las posibilidades de cierre y obertura son diversas, pero la situación más positiva para el 
desarrollo local parece ser cuando el sistema está cerrado en su organización y abierto en su estructura.

\section{Estudio de casos}

\subsection{Lérida}

Lérida es un municipio de 125.000 habitantes. En febrero de 2003 se inauguró la estación TAV donde ya existía la estación de ferrocarril clásica, ampliada. Cuando el tren esté en pleno funcionamiento situará la ciudad a cincuenta minutos de Barcelona y a dos horas de Madrid. El establecimiento del TAV en Lérida se tomó como un pretexto para mejorar la intermodalidad de la estación, las características urbanísticas del barrio y la economía de la ciudad.

La estación tendrá una accesibilidad mejorada a partir de diferentes actuaciones (una nueva pasarela, dos puentes nuevos, la reorganización del tránsito rodado, el traslado de la estación de autobuses a la estación, construcción de aparcamientos). Las mejoras en la accesibilidad tenían que permitir también mejorar la conexión de los «barrios norte» que habían quedado aislados, urbanística y socialmente, del Centro Histórico desde la construcción del trazado ferroviario a mediados del siglo XIX. Finalmente, las actuaciones de revitalización de los «barrios norte» incluyen la localización de nuevas actividades económicas como el nuevo centro de Congresos La Llotja.

La economía de la ciudad de Lérida está apoyada en dos grandes ejes. El primero se basa en los servicios de capitalidad (comerciales, empresariales) con un gran ámbito de influencia. Por ejemplo, es la segunda ciudad de Cataluña en número de ferias y congresos. El TAV tiene que servir para potenciar este sector, localizado principalmente en el Centro Histórico de la Ciudad. El segundo gran eje económico gira en torno al sector agroalimentario. El elevado número de hectáreas de regadío de la Plana de Lleida (150.000 ha.) y la industria relacionada al sector han favorecido la creación de un polo de investigación en la tecnología de los alimentos. Con la llegada del TAV, se ha planificado construir un Parque Tecnológico cerca de lo que tenía que ser la segunda estación de la ciudad, situada en el sur, pero por problemas con la administración central esta estación no se construirá.

\section{Red de actores locales}

La ciudad de Lérida se ha erigido como un actor colectivo con fuerza a partir del anuncio de la llegada del TAV. Es decir, que se ha activado una red de actores locales unidos por unos objetivos concretos de desarrollo para aprovechar las oportunidades que ofrecía la nueva gran infraestructura. Los actores que han formado el núcleo de la red son el Ayuntamiento, la Cámara de Comercio, la Diputación y la Universidad. Cada uno ha hecho una aportación específica al conjunto. El rol de liderazgo y de visión ha sido ejercido por el Ayuntamiento, incluso adelantándose a las propuestas de los actores supralocales. La Cámara de Comercio ha aportado una visión empresarial imprescindible para implicar el sector privado. La Diputación ha dado legitimidad territorial y política. Y la Universidad ha aportado conocimiento desde diversas vertientes, como la planificación, la economía o la agroalimentación. Este núcleo de actores ha conseguido implicar un amplio abanico de actores locales. Así pues, un número importante de actores han podido consensuar un proyecto de ciudad y unas líneas estratégicas para conseguirlo, aparte de algunas pequeñas desavenencias entre actores como el Ayuntamiento y la Diputación. En definitiva, se ha conseguido crear una dinámica positiva ante la llegada del TAV. 
Esto ha sido posible gracias a unos instrumentos de planificación como el Plan General de 1999, pero sobre todo el Pla de Dinamització de l'Alta Velocitat de les Terres de Lleida (www.lleidatav.org). Este Plan de naturaleza estratégica se ha estructurado a partir de grupos de trabajo específicos que han tratado los puntos clave a desarrollar con el TAV. Esta concreción temática ha permitido participar en cada ámbito los actores que estaban interesados. Aspectos del Plan como el marketing urbano han ayudado a extender los objetivos a la población y a participar activamente en su diseño.

\section{Ámbito territorial de la red de actores}

El límite territorial mejor definido de los actores que se han implicado en el proyecto del TAV es el municipal. A otros niveles, como el del área urbana o la región funcional, a pesar de la existencia de proyectos puntuales, no se ha conseguido una amplia implicación de los actores, como los ayuntamientos, los consejos comarcales, empresas. En todo caso, sí que existe una fuerte identidad con la ciudad de Lérida, por ejemplo a nivel de área urbana o, incluso, a escala de la región funcional, ya que existen unos recursos territoriales comunes. Eso facilitaría una activación futura de los agentes a estas escalas.

En cambio, a nivel provincial sí que encontramos la implicación de actores con el TAV, por ejemplo los relacionados con el turismo de los Pirineos. Sin embargo, más que actores del mismo sistema local de Lérida, podríamos considerar que los actores de otro sistema, el de los Pirineos, han mantenido unas relaciones de colaboración, han creado una alianza con los de Lérida para aprovechar unas oportunidades derivadas de la llegada del TAV. Es difícil pensar en un gran sistema local que tenga como ámbito toda la provincia o el área de influencia de Lérida. Por lo tanto, consideraremos que sistema local existe a nivel de la ciudad, limitado en el municipio.

\section{Cohesión funcional}

Así pues, podemos considerar que el SLoT de Lérida tiene un grado muy alto de cierre operativo (organización) y de apertura a los estímulos externos (estructura). Es decir que los actores que forman el sistema de Lérida han funcionado de manera conjunta y cohesionada y han mantenido los elementos propios de su sistema (agroalimentación, capitalidad); al mismo tiempo se han abierto a los estímulos que les venían del exterior en forma de la innovación que representaba el TAV (nueva accesibilidad, Parque Tecnológico, Centro de Congresos).

En términos de Maturana y Varela (1989) podemos decir que Lérida tiene una organización del sistema - es decir un «código genético» inmanente- donde hay dos elementos «geoestratégicos» que han sido adaptados a una estructura actual para que el sistema pueda seguir funcionando. La adaptación estructural ha sido posible gracias al revulsivo que ha supuesto la llegada del Tren de Alta Velocidad a Lérida.

El hecho de ser una ciudad relativamente alejada de la metrópoli barcelonesa puede haber influido en la reafirmación de la voluntad de capitalidad regional y de llevar a cabo un proyecto común. El TAV se ha visto como una forma de posicionarse en el territorio mejorando los recursos que tenía al alcance. El relativo alejamiento de los flujos más dinámicos del territorio catalán, que se sitúan más al litoral, y el peligro de mantenerse como una ciudad aislada, con una economía desfasada y con una tendencia al estancamiento, seguro de que han sido factores clave para conseguir estos grados de cierre y de apertura. Se podría defender que la ciudad estaba, antes de la llegada del TAV, en una cierta crisis. Quizás no era una crisis económica real, sino simplemente una percepción pesimista de la 
ciudad. Acciones del Plan de Dinamización como el Plan de Marketing han querido incidir en la recuperación de la autoestima y, por lo tanto, en la cohesión interna del sistema.

Las relaciones externas han tenido aspectos positivos y negativos. La fortaleza y cohesión del sistema han sido clave para abrirse a proyectos a una escala más vasta y para negociar con los actores supralocales (ministerio, GIF, Renfe) y pedir las modificaciones necesarias en el proyecto estatal de TAV. De esta manera se ha conseguido una estación TAV que potencia el centro de la ciudad y los proyectos económicos ligados a ésta. También ha habido relaciones externas a la hora de realizar la planificación estratégica del turismo o de mejorar la intermodalidad del transporte en la región.

Otro tipo de relaciones externas que se han dado, como hemos dicho antes, son con los agentes de lo que hemos llamado SLoT del Pirineo leridano. Se debe considerar una experiencia positiva el hecho que el TAV pueda servir para crear expectativas de desarrollo en un territorio más allá de la ciudad.

A pesar de los éxitos conseguidos ante el Estado, el actor local no ha sido lo bastante fuerte para conseguir una localización óptima para la segunda estación TAV de GardenyRufea, cuestión que puede considerar se como la pérdida de una oportunidad importante para la ciudad. El proyecto de ciudad se basa en la estructuración de dos polos complementarios que coinciden con las estaciones TAV. La falta de una de estas estaciones no impedirá que se desarrolle el Parque Tecnológico de Gardeny, pero éste no tendrá un posicionamiento territorial tan ventajoso como habría podido tener.

La debilidad de la ciudad como agente ante las instituciones supralocales del Estado no ha sido a causa de la falta de cohesión interna del sistema. Así pues, se tendría que culpar el desequilibrio existente entre el ámbito de planificación local y el supralocal en el contexto español, así como la falta de voluntad negociadora de los agentes estatales (Ministerio, GIF), que no permitieron la localización de la segunda estación por razones, en principio, no de carácter técnico sino político. Podríamos pensar también que la Generalitat de Catalunya, como agente de planificación regional, tampoco ayudó a fortalecer la capacidad del sistema local influyendo en las decisiones del agente estatal. La falta de alianza con el gobierno regional de la Generalitat habría agravado la debilidad del local.

Las razones de la falta de entendimiento entre los agentes no están en la poca capacidad de crear gobernanza, que hubiera estado a su alcance, sino en una problemática de enfrentamiento político. La falta de un marco de diálogo institucionalizado, en este contexto de poca voluntad política, puede haber agravado los desequilibrios en contra de un agente local débil, como esta ciudad intermedia. Esta debilidad podría aplicarse a todas las ciudades medias del país.

\subsection{Aviñón}

Aviñón (89.000 hab.) es la capital del Departamento de Vaucluse y pertenece a la Región de Provenza-Alpes-Costa Azul. La estación TAV se inauguró en el 2001 y sitúa la ciudad en treinta minutos de Marsella, a una hora de Lyón y a dos horas y media de París. La decisión sobre el trazado del TAV en la Provenza fue larga y conflictiva (1989-1995). Finalmente se decidió localizar la estación en las afueras de Aviñón, sin que coincidiera con la estación de tren histórica. Las dos estaciones se conectarán en el futuro con un tren ligero («virgule»).

El gobierno local no quería emplazar la estación en este lugar, llamado Courtine. Hubiera preferido localizarla al otro lado de la ciudad, cerca del aeropuerto y del nuevo Parque Tecnológico de la ciudad (Agropol), dedicado a la investigación agroalimentaria. Pero la conflictividad con el gobierno central y las grandes ciudades de la Región provocó una 
grave disputa interna entre el Ayuntamiento y los grupos ecologistas. Todo desembocó en el cambio de mayorías políticas en el gobierno de la ciudad y del Departamento. El nuevo gobierno no tenía ningún proyecto de desarrollo para el nuevo barrio de la estación -y ésta sería una de las causas que no se haya realizado todavía ninguna actuación alrededor de la estación TGV. Por este motivo, se encargó el proyecto de Courtine a una institución externa, sin demasiado liderazgo de los gobiernos locales. El resultado ha sido un Plan para el barrio donde se propone una «Cité de la formation» un - nuevo espacio con centros de seminarios, congresos, pedagoteca, etc., para la formación continua de trabajadores. También se aprovechará el barrio para la construcción de algunos hoteles y para equipamientos logísticos. En la investigación se defiende que este modelo de desarrollo, técnicamente impecable, no se puede considerar de tipo endógeno, ya que no fortalece plenamente el tejido económico existente.

\section{Red de actores locales}

Los sujetos locales activos son bastante numerosos y conforman una red local extensa, aunque con vínculos débiles, marcados a menudo por relaciones básicamente administrativas. La red está formada por el gobierno municipal de Aviñón, el resto de municipios de la aglomeración a partir de la COGA (Communauté d'Agglomération du Grand Avignon), el gobierno departamental de Vaucluse, los otros dos departamentos (Gard y Bouches du Rhône), la Cámara de Comercio, asociaciones de empresarios, asociaciones ambientalistas, CMT, CNR, etc.

El gobierno municipal no ha ejercido un rol de liderazgo y visión, cuando es el actor que tenía más posibilidades y capacidades de ejercer estas funciones. Tampoco lo ha hecho la COGA, un hecho explicable si tenemos en cuenta que se ha creado muy recientemente y no ha tenido casi tiempo de asentarse como una administración más del territorio. Ante esta situación el prefecto del Departamento parece erigirse como el líder (o al menos colíder) de la transformación de Courtine. El prefecto toma este rol como representante de la potente maquinaria administrativa francesa, que da al Departamento un peso político fuerte y unas capacidades técnicas de planificación importantes. Un ejemplo del coliderazgo y la importancia técnica del Departamento es que una sociedad pública como CITADIS, formada por el municipio de Aviñón y el Departamento de Vaucluse, es la encargada de planificar el Pôle d'affaires $T G V$.

Entre los sujetos que planifican los principales proyectos en torno al TGV hay colaboración, no hay conflictividad, pero es una colaboración casi pasiva. Muchas funciones, como la visión del futuro del barrio de Courtine, parecen estar delegadas a instituciones de planificación, como el AREP (organismo estatal), o a profesionales reconocidos (Desvigne, Perault, Zublena-Pochy-Koifman, Portzamparc, Wilmotte) que en realidad son actores externos. Quizás con este procedimiento las instituciones políticas acaban interiorizando los proyectos externos y el resultado es positivo. También se puede dar el caso de que el proyecto, técnicamente impecable, no se vea como propio por parte de la ciudad. En todo caso, la falta de visión explicaría también por qué el proyecto de planificación de Courtine se ha retrasado tanto en el tiempo. Diez años después de saberse la localización de la estación todavía no se ha materializado casi ninguno de los proyectos.

Los procesos de planificación han dado cierto juego a la participación de algunos agentes. El Estudio de Impacto ambiental permitió la participación de la población con la Encuesta de Utilidad Pública. El Plan de réference captó la opinión de los agentes económicos y sociales. Sin embargo, la implicación de la población en el nuevo proyecto de ciudad no ha sido elevada. Hay que destacar, sin embargo, que los procesos de participación, establecidos 
por ley, que se están llevando a cabo para redactar el nuevo PLU (Plan Local d'Urbanisme) pueden ayudar a crear una mayor dinámica interna en el futuro.

\section{Ámbito territorial de la red de actores}

Una de las características principales de la red de actores es su diversidad territorial, tanto respecto a las administraciones como para los agentes privados. Se encuentran las administraciones de tres departamentos y once municipios. Los agentes privados están representados por varias asociaciones ambientalistas (más bien desactivadas después del conflicto del TGV) del municipio de Aviñón, de Bouches du Rhône, de Gard, o diferentes agentes de los tres departamentos en torno a los empresarios de la asociación Grand Avignon.

La legislación francesa para la planificación supramunicipal y la individuación de diferentes áreas geográficas funcionales como ámbito de referencia (como el bassin de vie), aparte de la propia realidad urbana compleja, seguro que han ayudado a que los agentes del área urbana colaboren, a pesar de tratarse de límites administrativos diferentes.

Una consideración que puede hacerse es que quizás, al tratarse de una red de actores extensa territorialmente, hace más difícil la creación de vínculos fuertes. Aunque eso, en principio, no tiene por qué depender del ámbito territorial, sino de otras características como la falta de una identidad común fuerte a nivel de área urbana. El ámbito que podríamos considerar como propio del sistema local sería, por lo tanto, el del área urbana de Aviñón, aunque la mayoría de proyectos se producen dentro del municipio.

\section{Cohesión funcional}

Las características del sistema local territorial de Aviñón serían las de un sistema con un débil cierre operativo y con una apertura importante de actores y de proyectos exteriores. El resultado ha sido que los elementos económicos que caracterizaban Aviñón, como el turismo o el agroalimentación, han tenido transformaciones pequeñas o insignificantes, mientras que se ha apostado por una especialización no existente hasta ahora, abierta a atraer empresas del entorno.

En términos sistémicos, no es que haya variado la organización interna, sino que la llegada del TAV no ha provocado cambios cualitativos en ésta. No se han debilitado los recursos endógenos, simplemente no se han potenciado demasiado. En cambio, se ha introducido un elemento nuevo, la Ciudad de la formación, que responde a factores localizativos exógenos que se adaptan a una estructura externa.

La falta de cierre sería por causa tanto de factores externos como internos. Respecto a los externos, el más importante puede ser derivado de la conflictividad que se produjo en todo el proceso de planificación del TGV Méditerranée. Una de las vías de resolución del conflicto consistió en la ruptura de las alianzas entre los gobiernos locales y las asociaciones ambientalistas. Esta ruptura, si bien posibilitó la construcción final del trazado y la estación, habría tenido consecuencias negativas en el interior del sistema en forma de pérdida de confianza entre agentes. La conflictividad entre los agentes provocó, por ejemplo, el cambio de fuerzas políticas en el gobierno de la ciudad y en el Departamento.

Los factores internos serían los ya mencionados, la falta de visión y liderazgo de las instituciones políticas locales, tanto del gobierno municipal como del de la aglomeración (COGA). Especialmente, es la capacidad de liderazgo para la transformación lo que permite que un sistema esté unido. El liderazgo, sin embargo, tiene que ir precedido de una visión de la transformación. La visión de la ciudad del gobierno municipal, en vez de tomar el TAV como revulsivo para un nuevo proyecto de ciudad, ha consistido en un retorno a las 
características históricas de la ciudad, una visión que podríamos calificar de reactiva, causada quizás por el mismo conflicto de TGV. Una razón que puede explicar la falta de una visión innovadora de la ciudad es también la falta de crisis, económica o territorial, que no ha hecho movilizar la ciudad hacia un nuevo proyecto local. Otra razón podría provenir de la conflictividad vivida, que habría causado la falta de visión, de voluntad de cambio, hacia un nuevo proyecto de ciudad. Sin embargo, el débil cierre operativo del SLoT de Aviñón se vería atenuado por la existencia de una maquinaria técnica y política fuerte que ha permitido seguir adelante con la transformación de Courtine.

Esta transformación, sin embargo, ha sido protagonizada por actores externos y ha seguido una lógica de posicionamiento en un territorio amplio. De aquí la calificación que hacíamos de un sistema abierto a las influencias externas. Como hemos dicho, los planificadores de Courtine han sido la agencia AREP, dependiente de la SNCF, y un conjunto de profesionales del urbanismo y el paisajismo. El resultado ha sido un proyecto donde los principales agentes que han salido favorecidos son agentes «transversales», es decir agentes locales que tienen, o simplemente pertenecen a, una lógica externa.

\subsection{Novara}

Novara (102.000 hab.) es capital de provincia italiana y pertenece a la Región del Piemonte. La estación TAV fue inaugurada en febrero de 2006 y sitúa Turín a treinta minutos y Milán a quince minutos. En principio sólo tenía que haber una estación TAV en la ciudad, en la estación histórica, en el centro. Pero finalmente, por presiones de las ciudades mayores, se construirá una segunda estación en las afueras de Novara, en el by-pass, para evitar pasar por el centro de la ciudad.

Cuando se decidió realizar una única estación en el centro de Novara, la inversión se tomó como una oportunidad para remodelar todo el sistema ferroviario de la ciudad, para reestructurar urbanísticamente los barrios degradados en torno a la estación y para localizar cerca de la estación TAV los dos grandes equipamientos que simbolizan las especializaciones económicas de la ciudad: el Parque Tecnológico de la química y nuevos materiales, y el CIM (Centro de Intercambio de Mercancías). El Parque Tecnológico responde a la especialización histórica de la ciudad en la investigación y producción química — Novara era conocida como la «capital italiana de la química». El CIM responde a la posición territorial privilegiada en el centro de dos corredores viarios este-oeste y norte-sur, y a la proximidad al aeropuerto milanés de Malpensa. Las diferentes instituciones se unieron para planificar urbanística y estratégicamente las transformaciones de la ciudad.

La segunda estación se situará en las afueras y permitirá conectar ciudades como Turín directamente con Milán o con el aeropuerto de Malpensa, aunque restará potencialidad de transformación a la estación central. El gobierno local no estaba de acuerdo con esta decisión, pero no podía participar en las negociaciones donde se decidía su realización. El proyecto local de desarrollo quedaba, de esta manera, debilitado.

\section{Red de actores locales}

El anuncio de la llegada del TAV (o Alta Capacità) ha ayudado a la movilización de ciertos actores de la ciudad para promover una nueva etapa de desarrollo en Novara. La red de actores locales que se ha activado para aprovechar las ventajas del TAV ha acabado siendo bastante densa. En principio, fueron los actores institucionales los primeros a movilizarse, el gobierno municipal y el territorial. En torno a estos agentes se han adherido otros, como la Cámara de Comercio, diferentes empresarios implicados en la transformación física de 
la ciudad, empresas del sector químico, Universidad, asociaciones culturales y sociales, etc. Sin embargo, la participación de la ciudadanía ha sido más bien pobre.

La visión de la ciudad y la definición de estrategias han sido ejercidas básicamente por el gobierno municipal, a través de varios planes como el Pru, el Prusst (Piano di Riqualificazione Urbana e di Sviluppo Sostenibile del Territorio) o el Studio di fattibilià. Este actor es el que ha ido vehiculando las diferentes propuestas de configuración del nodo infraestructural y los proyectos relacionados con los actores supralocales, muchas veces tomando la iniciativa en la discusión. Las transformaciones han sido lideradas por el propio gobierno municipal y, en ciertos aspectos, por el gobierno territorial. En los aspectos de transformación física, el peso ha sido llevado por el municipio, mientras que en los de desarrollo económico, como el polo químico o el logístico, ha sido el gobierno territorial quien ha liderado las actuaciones (menos en casos como el turismo y ocio).

La dinámica entre los agentes ha sido positiva, aparte de ciertos enfrentamientos entre el gobierno municipal y el territorial, especialmente en la decisión de la localización de la segunda estación TAV y en la organización de la logística. Los instrumentos de planificación como el Prusst y el Plan Estratégico del Turismo parecen haber ayudado a crear una dinámica positiva entre los actores. Especialmente el Prusst ha servido para implicar en el proyecto al sector privado, necesario para la financiación que supone.

\section{Ámbito territorial de la red de actores}

La red de actores implicados en el TAV no ha traspasado las fronteras municipales. Debemos tener en cuenta que se trata de un municipio muy grande y con un peso demográfico y económico mucho más elevado que los municipios vecinos, y éstos miran con cierto recelo a la capital, al mismo tiempo que ésta se ve autosuficiente. Seguramente en el futuro deberán crearse alianzas, al menos, con el municipio vecino de Galliate, ya que compartirán la segunda estación TAV en línea. En todo caso, hay cierta identificación de los municipios de los alrededores con la capital, y ciertos recursos como la industria química o la logística están distribuidos por el área urbana.

En otros ámbitos como el provincial tampoco se han activado relaciones entre actores, aunque los actores del resto de la provincia, como los distritos industriales o el sector turístico de los lagos, en el norte, puedan imaginar las ventajas del TAV. El gobierno provincial tiene la intención de crear dinámicas de gobernanza a nivel provincial pero, en todo caso, estarán dirigidas a otros proyectos. Por lo tanto, debemos pensar que el SLoT se ha activado a escala municipal.

\section{Cohesión funcional}

Podemos calificar este SLoT como operativamente cerrado y abierto a las relaciones exteriores. Es decir, que el sistema ha conseguido reproducir y adaptar al contexto exterior aquellas especificidades económicas que lo habían caracterizado ya en el pasado, como el sector químico o los servicios de capitalidad territorial.

Esto ha sido posible gracias a la cohesión de la red de actores locales y al liderazgo de las transformaciones ejercidas por las instituciones locales para recuperar un rol de Novara en el territorio. En la posición territorial de la ciudad, dentro del ámbito metropolitano de Milán, se daba el peligro de perder la identidad y la actuación conjunta en favor de la integración dentro de las difusas dinámicas metropolitanas. El cierre interno garantiza una cierta identidad y la voluntad de desarrollar las especificidades propias de la ciudad en un contexto con tendencia a la configuración reticular. 
La apertura externa se ha producido a partir del desarrollo de ciertas especificidades que abarcan una escala amplia y sitúan Novara en el mapa del territorio italiano y europeo. En primer lugar se trata del polo científico y tecnológico de la química fina y nuevos materiales, que se erige como un centro de investigación a nivel internacional. En segundo lugar, se han reordenado los espacios y equipamientos de la logística para explotar una situación territorial nueva, donde Novara estará en el cruce de dos ejes ferroviarios este-oeste (entre Lyon y Trieste) y sur-norte (entre Génova y los túneles de Sempione y Gottardo). Tanto o más importante para la logística representa la apertura a la explotación de las posibilidades del aeropuerto de Malpensa.

Se puede concluir que la cohesión del sistema es bastante fuerte si no fuera por la desestabilización que supone la nueva estación en línea del TAV. Esta estación puede restar efectividad al proyecto local y al funcionamiento del nodo de transporte alrededor la estación central y a las potencialidades que se derivan, ya que no todos los trenes tendrán parada en la ciudad y la estación central. Eso no afectará tanto al sector logístico como a los otros elementos del proyecto local.

\section{Interpretación transversal de los casos}

Fruto de la lectura transversal de los casos de estudio podemos extraer algunas ideas referentes a la organización de los actores territoriales en torno a la nueva infraestructura ferroviaria.

\subsection{Relación multiescalar conflictiva}

Se pueden distinguir, en relación a los actores territoriales, tres escalas de actuación que corresponden a tres grupos de intereses o a tres niveles de proyectos para el Tren de Alta Velocidad. Se trataría de las escalas estatal, regional y local. A escala estatal, representada por el gobierno estatal y las empresas ferroviarias, el TAV está pensado principalmente para unir y articular las ciudades del país, dando preferencia a los trazados más directos y funcionales, aunque la tendencia es la construcción de estaciones en ciudades cada vez más pequeñas. A escala regional, representada por el gobierno regional y las grandes ciudades, los intereses predominantes son los de la articulación del territorio regional priorizando los intereses de conectividad de las ciudades grandes. Finalmente, a escala local, representada por los agentes locales, los intereses están en el posicionamiento de la ciudad media en el territorio y en el desarrollo local de la ciudad.

En este contexto, la escala local — la correspondiente a la ciudad media- es la que parte de una posición más débil, pero al mismo tiempo es la que puede comportar los procesos de desarrollo local en su interior. Por esta razón, y a causa de la existencia de varios intereses territoriales, los actores locales deben tomar un rol activo y propositivo, incluso de liderazgo, ante las otras escalas de decisión. Deben adelantarse, dentro de lo posible, a las propuestas de los actores supralocales.

Por otro lado, el proceso de planificación del TAV a menudo está marcado por una conflictividad importante entre los diversos agentes del territorio, locales y supralocales. Muchas veces esta conflictividad tiene una resolución deficiente, a partir de actitudes impositoras y de falta de diálogo, es decir, a partir de actitudes de government. Los comportamientos de government no son exclusivos sólo de las instituciones estatales, sino que los gobiernos regionales también pueden tomar a este modelo como forma de actuación.

La conflictividad no es, por sí misma, negativa. En cambio, sí que es negativa cuando está mal resuelta, porque comporta problemas en el desarrollo local (actitud reactiva, con- 
flictividad interna, falta de alianzas externas, debilidad del proyecto local). Por lo tanto, es necesario que los diversos agentes del territorio actúen a partir de unas dinámicas de diálogo y negociación, es decir actitudes de gobernanza.

\subsection{Red local de actores locales contenida}

La red de actores locales que lideran las transformaciones y que dibujan y pactan el proyecto local de desarrollo suelen corresponder a pocos actores y muy institucionalizados. Por un lado, el gobierno municipal es quien suele encabezar la red de actores implicados, especialmente, porque la estación se localiza dentro del municipio, dispone de las competencias en materia urbanística y tiene interiorizado - cuándo dispone de dirigentes con visión - un modelo económico y social de la ciudad con diferentes actuaciones concretas para llevarlo a cabo.

Otro actor presente es la «administración territorial» (según el país se trataría del gobierno provincial, el departamento, la diputación, etc.). Este actor da legitimidad territorial al proyecto, así como representatividad de los municipios más pequeños (que no suelen formar parte directamente de la red de actores que pactan el proyecto). La administración territorial suele mantener ciertas relaciones de conflictividad con el gobierno municipal, que no siempre son de carácter político —en Novara, por ejemplo, pertenecen al mismo partido-, sino que responden a una desconfianza mutua fruto de la competencia entre las dos administraciones.

Las Cámaras de Comercio y las Universidades (todas ellas presentes en las ciudades estudiadas) son los otros dos actores que se implican en el proyecto de transformación. Aparte de estos cuatro actores destacados, según el contexto se añaden un número más o menos elevado actores (empresas, asociaciones empresariales, otros municipios, ciudadanos, etc.), aunque, normalmente, la implicación de otros actores se hace difícil por los pocos mecanismos de participación de que se dotan las instituciones. La vía de participación puede ser más bien informal (como la participación en un plan estratégico) o más formal (como la encuesta de utilidad pública, o la elaboración de los PLU franceses).

Los caminos que pueden optimizar la creación de un proyecto local fuerte pasan por el establecimiento de una red de actores locales amplia, donde los agentes tengan que llevar a cabo diferentes roles. El actor local que parece estar mejor preparado para llevar a cabo el rol de visión y liderazgo de la transformación es el gobierno municipal -incluso si el proyecto tiene un alcance de área urbana. Es recomendable adherir a su alrededor a los otros agentes activos de la ciudad — como la Cámara de Comercio, la Universidad, empresarios, asociaciones culturales, asociaciones ambientalistas, gobierno territorial- y darles un rol activo, propositivo y especializado en el diseño del proyecto de ciudad y de las estrategias para conseguirlo. Los diversos agentes crean una forma óptima de relación si se distribuyen las funciones, como el desarrollo de ciertos sectores económicos (Cámara de Comercio, gobierno territorial), de complejos tecnológicos (Universidad), o de aspectos de la calidad de vida (ambientalistas). Esto es válido también para la participación ciudadana a través, por ejemplo, de ámbitos temáticos de participación. Para la implicación de la población en el proyecto parece útil, en último término, el trabajo para una nueva imagen e identidad de la ciudad. En el caso de participación en el diseño del proyecto de instituciones técnicas externas — recomendable por los conocimientos que aportan-, el liderazgo y la visión tienen que mantenerse siempre en las instituciones locales. 


\subsection{Conflictividad interna}

Otra constatación es el peligro de no resolver correctamente la conflictividad interna de los propios actores locales. Muchas veces, los conflictos que surgen entre las diversas escalas de administración (estatal, regional, local) se acaban resolviendo con decisiones difíciles de asumir por todos los agentes locales. De aquí surge una fuerte conflictividad interna que puede tener consecuencias: falta de cohesión, de soporte social, de visión. En dos de los casos estudiados, esta conflictividad ha sido la causa de un cambio de gobierno. Podríamos decir, en este sentido, que una consecuencia del TAV es que puede hacer perder unas elecciones.

Las posibles vías para resolver los conflictos internos parecen pasar por aumentar el grado de participación real en los proyectos y promover campañas de información ciudadana. El objetivo tiene que ser tanto la resolución de los conflictos internos que pueden surgir como el establecimiento de un acuerdo para llevar a cabo el proyecto local. Se entiende que la creación del proyecto local, antes que la definición de un escenario futuro, significa crear un proceso de diálogo entre los diversos actores de la ciudad interesados en el TAV. El conflicto de intereses que puede surgir no es negativo para la ciudad, sino que es necesario. Quizás la resolución de los conflictos se convierta en un proceso más lento, pero al final comporta beneficios. Se trata de conseguir, en definitiva, una dinámica positiva y creadora de la ciudad, no reactiva. Se debe abrir la participación a los diversos agentes de la ciudad, incluso a aquéllos que pueden estar enfrentados, ya que la participación se limita demasiado a menudo a las instituciones formales y a los agentes con intereses económicos.

\subsection{Dificultad para superar el ámbito municipal}

En términos territoriales, se constata la dificultad que surja un proyecto de ciudad que vaya más allá de los estrictos límites municipales de la ciudad. A pesar de los discursos supramunicipales, la realidad es que los municipios pequeños que limitan con la ciudad, y que muchas veces tienen elementos en común (polígonos industriales, barrios, estaciones, áreas de crecimiento...), no participan en el núcleo de la red de actores.

Por otro lado, se hace difícil pensar que esta dinámica cambie por sí sola si no hay iniciativas de las administraciones superiores que produzcan planificaciones de tipo supramunicipal con un contenido real. Es decir, con unas normas urbanísticas vinculantes, con dotación presupuestaria para sacar adelante proyectos comunes, etc.

El objetivo tendría que ser el establecimiento de una red de actores territorialmente más amplia con un proyecto local común basado en unas líneas estratégicas a nivel de área urbana.

\subsection{Crisis y capacidad proyectual de la ciudad}

Se da una estrecha relación entre la necesidad de la ciudad de dotarse de un nuevo proyecto local de desarrollo y el aprovechamiento del TAV para llevarlo a cabo. Esta afirmación tan obvia es una de las principales razones por las cuales unas ciudades sacan más provecho o menos de la nueva infraestructura.

Las ciudades que han estado gestando en los últimos años o decenios situaciones de crisis económica o de posicionamiento territorial son las que más se activan para sacar provecho de la nueva situación en la red de ciudades y las que más intentan aprovechar la nueva estación para llevar a cabo nuevos proyectos urbanos, tanto de tipo económico como urbanístico. Muchas veces, estos proyectos ya existían antes de la llegada del TAV, pero 
no se podían llevar a cabo por el elevado coste o por la falta de consenso entre los actores. El TAV, en estas ciudades, actúa como un revulsivo, como una chispa que enciende una nueva etapa histórica de la ciudad.

En cambio, hay ciudades que se encuentran históricamente en un momento dulce de su desarrollo, en una etapa de crecimiento económico, de buena posición territorial. Para estas ciudades el TAV se viene a sumar a los recursos que ya funcionan de la ciudad, pero no es visto como un revulsivo capaz de hacer cambiar el modelo de ciudad. Aunque debemos admitir que no suceden a menudo unas inversiones como las que supone una infraestructura como ésta y que, en la medida de lo posible, es muy recomendable aprovechar la oportunidad que representa para realizar cambios sustanciales o para reafirmar los ejes de desarrollo que ya funcionan en la ciudad.

\subsection{Dificultad para extender los beneficios del TAV en el territorio}

Uno de los objetivos de la construcción de una nueva infraestructura es la extensión de los beneficios en el resto del territorio, que no se concentren sólo en la ciudad donde se localiza la estación. Las ventajas del TAV pueden ser aprovechadas también por otras ciudades del territorio, por otros espacios que no están exactamente próximos a la ciudad, aunque a menudo es difícil que esto se produzca. Un ejemplo puede ser la movilización de actores del Pirineo que suscitó la estación TAV de Lérida. Los empresarios turísticos, apoyados por la Diputación de Lérida, vieron una oportunidad en el nuevo tren para promocionar este espacio —incluso la estación TAV se llamó «Lleida-Pirineus». Uno de los condicionantes para que otros territorios aprovechen el TAV es la correcta gestión de la intermodalidad, una cuestión ligada tanto a los aspectos técnicos como organizativos de los agentes implicados.

El establecimiento de alianzas con actores de otras ciudades y territorios próximos puede ser una política recomendable para extender los beneficios del TAV y para dar una actividad y una masa crítica suficiente a la estación TAV de la ciudad intermedia. Estas alianzas pueden ir acompañadas de un sistema de planificación física, estratégica y territorial a diferentes escalas: estación, municipio, área urbana y territorio.

\section{Conclusión final}

La metodología que se ha elaborado para estudiar la organización de los actores territoriales permite el análisis de los componentes subjetivos (de los sujetos, o implícitos) más importantes en el proceso de desarrollo local a partir del Tren de Alta Velocidad. Cabe recordar, pero, que es igualmente imprescindible el análisis de los factores objetivos (de carácter técnico, que también hemos llamado explícitos), aunque en este texto no se hayan descrito en profundidad por razones de temática y de extensión.

La metodología presentada cumple dos fases. En primer lugar, aborda el estudio de las características técnicas de la intervención y las organizativas de los agentes. En segundo lugar, a partir de esta información, interpreta el proceso de desarrollo local que ha aportado la implantación de la nueva infraestructura en el contexto urbano. El proceso de desarrollo realizado en las tres ciudades analizadas nos muestra diferencias organizativas en los contextos español, francés e italiano, diferencias que responden a esquemas de planificación más o menos jerárquicos, o más o menos formales.

Pero el análisis también nos muestra muchos elementos semejantes entre los casos de estudio. En todos ellos, la ciudad intermedia aparece como un actor muy débil en la relación con los otros actores implicados en la planificación del TAV. En primer lugar, la ciudad 
intermedia juega un papel residual en el proceso de decisión del TAV a escala multinivel, cuando, en realidad, es el actor mejor posicionado para liderar los procesos de desarrollo local. En segundo lugar, la reducida dimensión o masa crítica le dificulta el establecimiento de una red potente de actores locales con capacidad de influencia. En tercer lugar, y como consecuencia de las realidades anteriores, la ciudad intermedia es más sensible a la conflictividad local que surge con el proyecto de desarrollo. En cuarto lugar, se constata el problema de la superación de los límites municipales para establecer un ámbito mayor para el proyecto de desarrollo — una cuestión que se ha sido objeto de mejora en algún país como Francia, aunque sin demasiados resultados positivos por el momento. En quinto lugar, se observa la relación entre el estado de crisis de la ciudad y la voluntad de la misma de auto-reinventarse con un nuevo proyecto de desarrollo. Finalmente, y no por ser el último es menos importante, se constanta también la dificultad que otros territorios y ciudades próximas puedan aprovechar la llegada del TAV par su propio desarrollo, es decir, que se ve mermada la capacidad de crear desarrollo regional.

Probablemente, no todos los problemas que afectan a la ciudad intermedia tienen solución, o la tienen en diferentes grados. Aún y así, muchas de las problemáticas provienen de la falta de una correcta organización de los actores locales. Podemos distinguir, además, los dos ámbitos donde esta organización es deficiente: en la relación multinivel entre los diferentes actores, y entre los propios actores locales. Tanto en un ámbito como en el otro, pueden producirse mejoras organizativas en la línea de lo que muchos autores llaman el paso de un proceso de gobierno (government) a uno de gobernanza (governance) (por ejemplo, Governa, 2002). De esta manera, podemos hablar de acciones dirigidas a una gobernanza externa y una gobernanza interna. Para una correcta gobernanza externa, las instituciones supralocales deben actuar a partir de dinámicas de diálogo y negociación. También deben favorecer instrumentos de planificación adecuados para la gestión de las áreas urbanas de las ciudades intermedias y la gestión de la intermodalidad a escala regional. Para la gobernanza interna, los actores locales deben seguir directrices a favor de: tomar un rol activo y propositivo ante las otras escalas de decisión; establecer una red de actores locales amplia, donde los agentes protagonizen diferentes roles; aumentar el grado de participación real de los diferentes agentes de la ciudad a los proyectos locales; y aprovechar la oportunidad que representa el TAV para realizar cambios sustanciales, o para reafirmar los ejes de desarrollo, en el modelo de ciudad.

\section{Bibliografía}

BAGNASCO, A; LE GALÈS, P. (1997): «Introduction. Les villes européennes come société et come acteur», en BAGNASCO, A; LE GALÈS, P., (a cura de), Villes en Europe. La Découverte. Paris, pp. 7-43.

BELLET, C. (coord.) (2000): Les oportunitats del tren d'alta velocitat a Lleida. Pagès Editors. Lleida.

BERG, L. VAN DEN; DER MEER, J VAN; POL, P. (2003): «Organizing Capacity and Social Policies in European Cities», en Urban Studies, vol. 40, $\mathrm{n}^{\circ}$ 10, pp. 1.959-1.978.

BERG, L. VAN DEN; POL, P. (1997): «The organising capacity of metropolitan regions», en Environment and Planning C: Government and Policy, vol. 15, pp. 253-272.

BERG, L. VAN DEN; POL, P. (1998): The European high-speed train-network and urban development: experiences in fourteen European urban regions, European institute for comparative urban research, Ashgate.

BURMEISTER, A.; COLLETIS-WAHL, K. (1997): «Les interactions productio-transport-espace: quelle(s) logique(s) de proximité(s)?», en Revue d'Economie Regionale et Urbaine, $\mathrm{n}^{\circ}$ 3, 1997, pp. 363-3861. 
CASTELLS, M. (1997): La era de la información. Economia, Sociedad y cultura, Vol. 2 El Poder de la Identidad. Alianza editorial. Madrid.

CONTI, S. (1996): «Il linguaggio dei sistemi», en Conti, Sergio, Geografia Economica: teorie e metodi. UTET Libreria. Torino, pp. 181-233.

DEMATTEIS, G. (1985): Le metafore de la Terra. La geografia umana tra mito e scienza. Feltrinelli. Milano.

DEMATTEIS, G. (1994): «Possibilità e limiti dello svilupo locale», en Svilupo locale, nº 1, pp. 10-30.

DEMATTEIS, G. (1995): Progetto implicito. Il contributo della geografia umana alle scienze del territorio. Franco Angeli. Milano.

DEMATTEIS, G. (2003): «Il modello SLoT come strumento di analisi dello sviluppo locale», em Rossignolo, C.; Imarisio, C. (a cura de), SLoT quaderno 3. Una geografia dei luoghi per lo sviluppo locale. Approcci metodologici e studi di caso. Baskerville. Bologna, pp. 13-27.

FELIU, J. (2005): Les grans infrastructures $i$ el desenvolupament local de les ciutats mitjanes. El Tren d'Alta Velocitat a les ciutats de Lleida, Avinyó i Novara. Directora: Margarida Castañer. Girona: Universitat de Girona. Institut de Medi Ambient.

FELIU, J. (coord.) (2006a): Estudio marco de los efectos del tren de alta velocidad en la ciudad intermedia. Interreg III A- Evaluación del impacto socioeconómico del tren de alta velocidad (TAV) en el espacio transfronterizo. Girona.

FELIU, J. (2006b): El tren d'alta velocitat. Desenvolupament local a Lleida, Avinyó i Novara. Cambra de Comerç de Girona-Càtedra Empresa de la Universitat de Girona, Girona,.

FOERSTER, H. VON (1985): «Cibernetica ed epistemologia: storia e prospettive», en BOCCHI, G.; CERUTI, M., La sfida della complessità. Feltrinelli. Milano, pp. 112-140.

FUENTE, R. S de la. et alii. (2006): «Analisis de las consecuencias territoriales del AVE en ciudades pequenas: Ciudad Real y Puertollano», en Estudios Territoriales, n²60, pp. 199-230.

GOVERNA, F. (1997): Il milieu urbano. L'identità territoriale nei processi di svilupo. Franco Angeli. Milano.

GOVERNA, F. (2001): «Ripensare il raporto infrastutture/territorio. Il valore aggiunto territoriale delle infrastrutture di transpoto», en DEMATTEIS, G.; GOVERNA, F. (a cura di) Contesti locali e Grandi Infrastrutture. Politiche e progetti in Italia e in Europa. Franco Angeli. Milano, pp. 27-48.

GOVERNA, F. (2002): «Del government a la governance. En canvi de les formes i les modalitats de l'acció col-lectiva en el camp urbà i territorial», en Documents d'Anàlisi Geogràfica, nº 41, pp. 43-62.

JOIGNAUX, G. (1997): «L'approche des relations entre infrastructures et territoires: retours sur la théorie et les méthodes», en Burmeister, A.; Joinaux, G. Infrastructures de transport et territoires. L'Harmattan. Paris, pp. 17-38.

LOLIVE, J. (1999): Les contestations du TGV Méditerranée: Projet, controverse et espace public. col.logiques politiques. Harmattan. París.

MAGNAGHI, A. (a cura de) (1990): Il territorio dell'abitare. Lo sviluppo locale come alternativa strategica. Franco Angeli. Milano.

MAGNAGHI, A. (2000): Il progetto locale. Bollati Boringhieri. Torino.

MARTÍ-HENNEBERG, J. (2000): «Un balance del tren de alta velocidad en Francia. Enseñanzas pera el caso español», en Ería, nº 52, pp.131-143.

MATURANA, H.; VARELA, F. (1989): El Árbol del conocimiento: las bases biológicas del conocimiento humano. Debate. Madrid.

MIRALLES, C. (2002): «Transporte y territorio urbano: del paradigma de la de la causalidad al de la dialéctica», en Documents d'Anàlisi Geogràfica, UAB-UdG, nº 41, pp. 107-120.

OFFNER, J. M. (1993): «Les «effets structurants» du transport: mythe politique, mystification scientifique», en L'Espace Geographique., nº 3, p. 233-242.

PLASSARD, F. (1997): «Les effets des infrastructures de transport, modèles et paradigmes«, en BURMEISTER, A.; JOINAUX, G. Infrastructures de transport et territoires. L'Harmattan. Paris, pp. 39-54. 
PLASSARD, F. (2003): Transport et territorire. La Documentation française. Paris.

PUCCI, P. (1996): I nodi infrastrutturali: luoghi e non luoghi metropolitani. Franco Angeli. Milano.

POL, P. (2002): A renaissance of stations, railways and cities: economic effects, development strategies and organisational issues of European HST stations. TRAIL thesis series. Erasmus Universiteit Rotterdam.

RABIN, G. (2003): Villes et grande vitesse. La révolution ferroviaire en France et Allemagne. Mardaga.

RIBALAYGUA, C. (2005), «Alta Velocidad y ciudad: estrategias de incorporación de las nuevas estaciones periféricas francesas y españolas», Colección CIU, 44, Instituto Juan Herrero, Universidad Politécnica de Madrid. Madrid. 